International Journal of Pure and Applied Mathematics

Volume $90 \quad$ No. $4 \quad 2014,387-406$

ISSN: 1311-8080 (printed version); ISSN: 1314-3395 (on-line version)

url: http://www.ijpam.eu

doi: http://dx.doi.org/10.12732/ijpam.v90i4.1

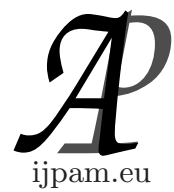

\title{
ANALYSIS OF SOME DIFFERENCE SCHEMES USED IN NUMERICAL SIMULATION OF PRODUCTION/INJECTION WELLS
}

\author{
Igor Savin ${ }^{1}$, Boris Kunin ${ }^{2} \S$ \\ ${ }^{1,2}$ Department of Mathematical Science \\ University of Alabama \\ Huntsville, 301 Sparkman Drive, Huntsville, AL 35899, USA
}

\begin{abstract}
Asymptotic approximation/truncation error of a numerical scheme commonly used in petroleum engineering is analyzed. It is shown that the scheme has a (small) non-removable asymptotic error. A modification that eliminates the error from the scheme is found.
\end{abstract}

AMS Subject Classification: 65N06, 65N12, 65N80, 65Z99

Key Words: reservoir simulations, well modelling, flows in porous media, filtration

\section{Introduction}

In this paper, we consider the problem of the steady systematic pressure filtration of a liquid towards a borehole. It is assumed that the filtration occurs through a homogeneous bounded strata with constant viscosity and diffusion coefficients. The problem reduces to finding the solution of the Laplace equation in a doubly-connected domain whose outer boundary is the contour of the domain of filtration. In practice, the dimensions of the domain of filtration $\left(\sim 10^{3} \mathrm{~m}\right)$ are much greater than the dimensions of the borehole $\left(\sim 10^{-1} \mathrm{~m}\right)$. Therefore, in the solution of this problem by a grid method, it is not advis-

Received: April 9, 2013

(c) 2014 Academic Publications, Ltd.

${ }^{\S}$ Correspondence author url: www.acadpubl.eu 
able to approximate the domain of filtration by a grid domain. Indeed, a large number of mesh points would be required after taking into account the size and shape of the borehole. So, in a numerical solution of this problem it is common to view the dimensions of the borehole as negligible, to treat the borehole as a point source, and assign to it the flow rate of the real source. (In view of the comparative smallness of the size of the borehole, the flow in its neighborhood may be regarded as radial.) Such an approximation gives a good accuracy.

The situation becomes more complicated if, on the contour of the borehole, not the flow rate, but the head (pressure), is given (Dirichlet conditions). It follows from [1] that in this case it is impossible to contract the borehole to a point. The grid approximation of the problem with the assignment of a known value of the solution to the nodal borehole does not lead to the desired result either. Thus, there arises the question of how to approximate the "problem of a borehole with a given head." Two basic approaches are used in solving problems where such singularity occurs. One is based on "coefficient adjustment" method and the other one makes use of clustering grids (special mesh refinement). In this paper we investigating the first one.

In other words, when modeling a well in numerical reservoir simulation, the problem of finding the pressure of the well being modeled is characterized by the difficulty of grid resolution. The size of the filtration area is always much greater than the radius of the well. Accordingly, the grid blocks resulting from the discretization of the domain can never be made small enough to resolve the radius of the well due to limitations of processor capability. Additionally, it is well known that the solution of such a boundary value problem cannot be altered or changed in any way at any point, because such a contravention results in the problem becoming overdetermined. Therefore, the grid cannot be resolved fine enough. The completely determined solution in this domain cannot be altered to accommodate the limitation resulting from the difference in well radius and the dimensions of the grid blocks.

For the domain $D=\left\{(x, y): R>\left(x^{2}+y^{2}\right)^{\frac{1}{2}}>\varepsilon\right\}$, where $0<\varepsilon \ll R$ is a small parameter, we will consider the boundary-value problem

$$
\begin{aligned}
& \Delta u=0 \text { in } D, \\
& \left.u\right|_{r=\varepsilon}=u_{0}=\text { const, } \\
& \left.u\right|_{r=R}=u_{1}=\text { const }
\end{aligned}
$$

where $r=\left(x^{2}+y^{2}\right)^{\frac{1}{2}}$ and $\Delta$ is the Laplace operator. (We placed the well of radius $\varepsilon$ at the origin.) 
The exact analytical solution of the problem (1) - (3) is known:

$$
u(x, y)=u(r)=u_{1}+\frac{\left(u_{0}-u_{1}\right) \ln \left(\frac{R}{r}\right)}{\ln \left(\frac{R}{\varepsilon}\right)}, \quad \varepsilon<r \leq R .
$$

Several authors have considered discretization of this problem. In [2][10], the authors focused on two-dimensional flow and observed that for a well inside a grid block, the block pressure is not an average pressure. Examination of the numerical solution for Laplace's equation using the repeated five-spot pattern/stencil showed that the numerical solution for pressure plotted versus radius exhibits a linear trend. The results from [2],[5]-[7],[10] achieve a better description of well block pressure by modifying the approximation in terms 'equivalent well radius'. The authors of [4] approached the problem using the five-point pattern for a well located at a node, and introduced a correction factor for each used approximation. These correction factors are shown to greatly improve the determination of the pressures by the difference method. In [5], it was stated that the calculated pressure for a well is the pressure of the node in which it is located, and that the pressure should be compared with the average pressure in that portion of the reservoir represented by the node. The authors of [6] derived a solution for systems where the well block is a corner block or an edge block, and also for off-center wells within a grid block. These results are a modification of approximations from [2] for an interior grid block with a well located at the center of a block. The results obtained in [7] are similar to those of [2], except that in [7] no restriction was placed on the position of the well within a grid block. This approach was summarized in [11]-[15]

In [4], [8], and [9], the differential problem (1) - (3) is approximated with an 'adjustment' method in two different ways which yield varying accuracies. The coefficients of the difference equations which approximate the differential problem are constructed (adjusted), taking into account the singularity of the solution in the neighborhood of the well. In [4],[9] the authors derive the coefficients of difference schemes by calculating selected fluxes through the contours of grid blocks around the well. In [8], they also derive difference schemes using an explicit isolation of the logarithmic singularity of the solution. The derivations are given in the Appendix. We show in the Appendix that heuristical approaches lead to identical difference schemes. We rewrite these two schemes from $[4],[9],[8]$ below in a form that is more convenient for analysis. As one of the outcomes of the analysis, we indicate that the approximations proposed in [2]-[10] and included in the contemporary handbooks [11]-[15] have nonremovablle asymptotical error or, in other words, they are not asymptotically 
exact.

\section{Approximations and Their Analysis}

In this section, two finite difference approximations of problem (1) - (3) on the mesh $\Omega$ will be formulated and compared with exact solution. The first of the two approximations to be considered is the simpler one.

Introduce a square grid $\Omega=\{(x, y): x=i h, y=j h ; i, j \in \mathbb{Z}\}$. The well is at $(0,0)$. For a function $w(x, y)$, put $w_{i j}=w\left(x_{i}, y_{j}\right)$. The grid Laplacian, for which two modifications will be considered, is

$$
\Lambda w_{m, n} \equiv \frac{1}{h^{2}}\left(w_{m+1, n}+w_{m-1, n}+w_{m, n+1}+w_{m, n-1}-4 w_{m, n}\right) .
$$

The first modification involves the immediate neighborhood of $(0,0)$, the second one involves a wider neighborhood. Specifically, let us introduce the sets

$$
\begin{aligned}
& \mathrm{L}=\{(-1,0),(1,0),(0,-1),(0,1)\}, \\
& \mathbf{F}=\{(-1,-1),(1,1),(1,-1),(-1,1)\} .
\end{aligned}
$$

For a node $(i, j)$, let $p_{i j}=p(i h, j h)$, whereas $p=(x, y)$ will stand for a generic point in the plane or grid $\Omega$, and let $r=r(p)=\left(x^{2}+y^{2}\right)^{1 / 2}$. Also, let $\omega=\{p \in$ $\Omega: r(p)<R\}$.

The modification \#1 of the grid Laplacian contains a parameter $k$ and is given by

$$
\Lambda_{1} \equiv \Lambda+\sum_{L \in \mathrm{L}} \delta_{h}\left(p-p_{L}\right) M_{L}, \quad p_{L} \equiv p_{i, j} \quad \text { for }(i, j) \in \mathbf{L}
$$

where

$$
\delta_{h}(p)=\delta_{h}(i, j)= \begin{cases}\frac{1}{h^{2}} & \text { for } i=j=0, \\ 0 & \text { for } i^{2}+j^{2} \neq 0,\end{cases}
$$

is a grid analog of Dirac's delta function, and the operators $M_{L}, L \in \mathrm{L}$, are defined by ( $\mathrm{k}$ is a parameter)

$$
\begin{aligned}
& M_{(1,0)} v_{1} \equiv(k-1) v_{1_{0,0}}+(1-k) v_{1_{1,0}}, \\
& M_{(-1,0)} v_{1} \equiv(k-1) v_{1_{0,0}}+(1-k) v_{1_{-1,0}}, \\
& M_{(0,-1)} v_{1} \equiv(k-1) v_{1_{0,0}}+(1-k) v_{1_{0,-1}}, \\
& M_{(0,1)} v_{1} \equiv(k-1) v_{1_{0,0}}+(1-k) v_{1_{0,1}} .
\end{aligned}
$$

One can easily verify that at every node from $L$ the sum 
$\sum_{L \in \mathrm{L}} \delta_{h}\left(p-p_{L}\right) M_{L}$ has exactly one non-zero term, which produces the modification of $\Lambda$ in question. Evidently, the sum equals zero at every other node. For example, at $(i, j)=(1,0)$,

$$
\begin{aligned}
\Lambda_{1} w=\quad & \frac{1}{h^{2}}\left(\left(w_{2,0}-w_{1,0}\right)-k\left(w_{1,0}-w_{0,0}\right)\right. \\
& \left.+\left(w_{1,1}-w_{1,0}\right)-\left(w_{1,0}-w_{1,-1}\right)\right) .
\end{aligned}
$$

The resulting approximation \#1 of (1)-(3) becomes

$$
\begin{aligned}
& \Lambda_{1} v_{1}(p)=0, \quad p \neq p_{0,0}, \quad v_{1}\left(p_{0,0}\right)=u_{0}, \quad p \in \omega, \\
& v_{1}(p)=u(p), \quad p \in \Omega \backslash \omega .
\end{aligned}
$$

where $u(r)$ is defined by (4).

The modification \#2 of the grid Laplacian contains parameters $k, b$ and is given by

$$
\begin{aligned}
& \Lambda_{2} \equiv \Lambda+\sum_{F \in \mathrm{F}} \delta_{h}\left(p-p_{F}\right) B_{F}+\sum_{L \in \mathrm{L}} \delta_{h}\left(p-p_{L}\right) N_{L}, \\
& p_{L} \equiv p_{i, j} \quad \text { for }(i, j) \in \mathrm{L}, \quad p_{F} \equiv p_{i, j} \quad \text { for }(i, j) \in \mathbf{F},
\end{aligned}
$$

where the operators $B_{F}, F \in \mathrm{F}$, and $N_{L}, L \in \mathrm{L}$, are defined by (b and k are parameters)

$$
\begin{aligned}
& B_{(1,1)} v_{2} \equiv(b-1) v_{2_{0,1}}+(1-b) v_{2_{1,1}}+(b-1) v_{2_{1,0}}+(1-b) v_{2_{1,1}}, \\
& B_{(-1,1)} v_{2} \equiv(b-1) v_{2_{0,1}}+(1-b) v_{2_{-1,1}}+(b-1) v_{2_{-1,0}}+(1-b) v_{2_{-1,1}}, \\
& B_{(-1,-1)} v_{2} \equiv(b-1) v_{2_{-1,0}}+(1-b) v_{2_{-1,-1}}+(b-1) v_{2_{0,-1}}+(1-b) v_{2_{-1,-1}} \text {, } \\
& B_{(1,-1)} v_{2} \equiv(b-1) v_{2_{0,-1}}+(1-b) v_{2_{1,-1}}+(b-1) v_{2_{1,0}}+(1-b) v_{2_{1,-1}} . \\
& N_{(-1,0)} v_{2} \equiv \quad(b-1) v_{2-1,1}+(1-b) v_{2_{-1,0}}+(b-1) v_{2-1,-1} \\
& +(1-b) v_{2_{-1,0}}+(k-1) v_{2_{0,0}}+(1-k) v_{2_{-1,0}} \text {, } \\
& N_{(0,-1)} v_{2} \equiv \quad(b-1) v_{2_{-1,-1}}+(1-b) v_{2_{0,1}}+(b-1) v_{2_{1,-1}} \\
& +(1-b) v_{2_{0,-1}}+(k-1) v_{2_{0,0}}+(1-k) v_{2_{0,-1}} \text {, } \\
& N_{(1,0)} v_{2} \equiv(b-1) v_{2_{1,-1}}+(1-b) v_{2_{1,0}}+(b-1) v_{2,1} \\
& +(1-b) v_{2_{1,0}}+(k-1) v_{2_{0,0}}+(1-k) v_{2_{1,0}} \text {, } \\
& N_{(0,1)} v_{2} \equiv(b-1) v_{2-1,1}+(1-b) v_{2_{0,1}}+(b-1) v_{2,1}
\end{aligned}
$$




$$
+(1-b) v_{2_{0,1}}+(k-1) v_{2_{0,0}}+(1-k) v_{2_{0,1}} .
$$

The resulting approximation \#2 of (1)-(3) becomes

$$
\begin{aligned}
& \Lambda_{2} v_{2}(p)=0, \quad p \neq p_{0,0}, \quad v_{2}\left(p_{0,0}\right)=u_{0}, \quad p \in \omega \\
& v_{2}(p)=u(p), \quad p \in \Omega \backslash \omega,
\end{aligned}
$$

Both approximations appear in literature, where both $\Lambda_{1}$ and $\Lambda_{2}$ appear as modifications of various terms in $\Lambda$ in a vicinity of the well. In Appendix A, we describe the nature of the modifications as well, as physical reasoning used (in the literature) to come up with the values of the parameters $k, b$.

In what follows, we analyze approximation \#1 for being asymptotically exact. We show that there is a unique value of $k$ for which exactness occurs and we point out that this value is different from various heuristic ones used in the literature (including the latest petroleum engineering handbooks).

As for the approximation \#2, we show that the heuristic literature values of $k$ and $b$ result in an approximation that is not asymptotically exact.

In the approach we take, we need some information about the Greens function of the operator $\Lambda$.

\subsection{Green's Function of the Discrete Laplace Operator}

The Greens function $\mathcal{E}\left(x_{i}, y_{j}\right)$ of the operator $\Lambda$ is the solution of the following problem

$$
\begin{array}{r}
\Lambda \mathcal{E}(p)=-\delta_{h}(p), \quad p \in \Omega, \\
\mathcal{E}\left(p_{0,0}\right)=0, \quad \lim _{r \rightarrow \infty} \frac{\mathcal{E}}{r}=0, \quad r=\left(x^{2}+y^{2}\right)^{1 / 2} .
\end{array}
$$

The uniqueness of the solution of problem (12) is proven in [16]. It is known [16], [17], that

$$
\mathcal{E}(x, y)=\frac{1}{(2 \pi)^{2}} \int_{-\pi}^{\pi} \int_{-\pi}^{\pi} \frac{\cos (x \xi / h) \cos (y \eta / h)-1}{4\left(\sin ^{2}(\xi / 2)+\sin ^{2}(\eta / 2)\right)} d \xi d \eta
$$

If $r=O(1)$ the asymptotic formula

$$
\mathcal{E}(x, y)=\frac{1}{2 \pi}\left(\ln \frac{h}{r}-\frac{3}{2} \ln 2-\gamma\right)+e(h, r, \varphi),
$$

holds for $\mathcal{E}(x, y)$, where $\gamma$ is Euler's constant, $\varphi=\arctan (y / x)$, 
Table 1: Exact (right upper part) and approximate (left lower part) values of point source function $\mathcal{E}\left(x_{i}, y_{j}\right)$

\begin{tabular}{|c|c|c|c|c|c|c|}
\hline $\mathbf{j} \backslash \mathbf{i}$ & $\mathbf{0}$ & $\mathbf{1}$ & $\mathbf{2}$ & $\mathbf{3}$ & $\mathbf{4}$ & $\mathbf{5}$ \\
\hline $\mathbf{0}$ & $0.00000 \backslash 0$ & $\frac{1}{4}$ & $1-\frac{2}{\pi}$ & $\frac{17}{4}-\frac{12}{\pi}$ & $20-\frac{184}{\pi}$ & $\frac{401}{4}-\frac{940}{\pi}$ \\
\hline $\mathbf{1}$ & 0.25000 & $0.31831 \backslash \frac{1}{\pi}$ & $-\frac{1}{4}+\frac{2}{\pi}$ & $-2+\frac{23}{\pi}$ & $-\frac{49}{4}+\frac{40}{\pi}$ & $-70+\frac{3323}{15 \pi}$ \\
\hline $\mathbf{2}$ & 0.36338 & 0.38662 & $0.42441 \backslash \frac{4}{3 \pi}$ & $\frac{1}{4}+\frac{2}{3 \pi}$ & $3-\frac{118}{15 \pi}$ & $\frac{97}{4}-\frac{1118}{15 \pi}$ \\
\hline $\mathbf{3}$ & 0.43028 & 0.44038 & 0.46221 & $0.48808 \backslash \frac{23}{15 \pi}$ & $-\frac{1}{4}+\frac{12}{5 \pi}$ & $-4+\frac{499}{35 \pi}$ \\
\hline $\mathbf{4}$ & 0.47700 & 0.48239 & 0.49596 & 0.51394 & $0.53355 \backslash \frac{176}{105 \pi}$ & $\frac{1}{4}+\frac{20}{21 \pi}$ \\
\hline $\mathbf{5}$ & 0.51290 & 0.51625 & 0.52530 & 0.53819 & 0.55315 & $0.56892 \backslash \frac{563}{315 \pi}$ \\
\hline
\end{tabular}

and $e(h, r, \varphi)=O\left(h^{2} / r^{2}\right)$ [18],[19].

Table 1 (from [20]) gives the values of $\mathcal{E}\left(x_{i}, y_{j}\right)$ for $0 \leq i, j \leq 5$.

In the next two subsections, we will find solutions of difference schemes 9 and 11 by representing the solutions as combinations of Greens functions and a constant.

\subsection{Approximation \#1 (Analysis)}

To examine the approximation (9), let us let us seek the solution in the form

$$
v_{1}(p)=\alpha_{1}+\beta_{1} \mathcal{E}(p)+\gamma_{1} \sum_{L \in \mathrm{L}} \mathcal{E}\left(p-p_{L}\right),
$$

where $\alpha_{1}, \beta_{1}$ and $\gamma_{1}$ are constants to be determined.

Applying the operator $\Lambda_{1}$ and the corresponding boundary conditions to equation (14), a nonsingular linear algebraic system of three equations with respect to $\alpha_{1}, \beta_{1}$ and $\gamma_{1}$ emerges:

$$
\left(\Lambda_{1} v_{1}(p)\right)_{(1)} \equiv-\gamma_{1}+\beta_{1}\left(M_{L} \mathcal{E}(p)\right)_{(1)}+\gamma_{1} \sum_{L \in \mathrm{L}}\left(M_{L} \mathcal{E}\left(p-p_{L}\right)\right)_{(1)}=0
$$




$$
\begin{aligned}
& \alpha_{1}+4 \mathcal{E}\left(p_{1,0}\right) \gamma_{1}=u_{0}, \\
& \alpha_{1}+\left(\beta_{1}+4 \gamma_{1}\right) \mathcal{E}(R)=u_{1} .
\end{aligned}
$$

The explicit expressions for terms $\left(M_{L} \mathcal{E}(p)\right)_{(1)}$ and $\left(M_{L} \mathcal{E}\left(p-p_{L}\right)\right)_{(1)}$ can be written as

$$
\begin{aligned}
& \left(M_{L} \mathcal{E}(p)\right)_{(1)}=(k-1) \mathcal{E}_{00}+(1-k) \mathcal{E}_{10}, \\
& \sum_{L \in \mathrm{L}}\left(M_{L} \mathcal{E}\left(p-p_{L}\right)\right)_{(1)} \\
& =(k-1) \cdot 4 \mathcal{E}_{10}+(1-k)\left(\mathcal{E}_{20}+\mathcal{E}_{00}+2 \mathcal{E}_{11}\right) .
\end{aligned}
$$

Substituting (16) and (17) into (15) and taking into account the values for $\mathcal{E}\left(p_{i, j}\right) \equiv \mathcal{E}\left(x_{i}, y_{j}\right) \equiv \mathcal{E}_{i j}$ from Table 1 , we can rewrite (15) in the form:

$$
\begin{aligned}
-\gamma_{1}-\frac{1}{4} \beta_{1}(1-k) & =0 \\
\alpha_{1}-\gamma_{1} & =u_{0}, \\
\alpha_{1}+\left(\beta_{1}+4 \gamma_{1}\right) \mathcal{E}(R) & =u_{1} .
\end{aligned}
$$

The solution to this system is

$$
\begin{aligned}
& \alpha_{1}=u_{1}-\left(u_{0}-u_{1}\right) \cdot \frac{\mathcal{E}(R)}{\frac{1-k}{4 k}-\mathcal{E}(R)}, \\
& \beta_{1}=\left(u_{0}-u_{1}\right) \frac{\frac{1}{k}}{\frac{1-k}{4 k}-\mathcal{E}(R)}, \\
& \gamma_{1}=\left(u_{0}-u_{1}\right) \frac{\frac{k-1}{4 k}}{\frac{1-k}{4 k}-\mathcal{E}(R)} .
\end{aligned}
$$

For $\mathcal{E}(R)$ we have from $(13)$

$$
\mathcal{E}(R)=\frac{1}{2 \pi}\left(\ln \frac{h}{R}-\frac{3}{2} \ln 2-\gamma\right)+O\left(\frac{h^{2}}{R^{2}}\right) .
$$

Substituting $\alpha_{1}, \beta_{1}, \gamma_{1}$ and $\mathcal{E}(R)$ into the linear combination (14), and bearing in mind that

$$
4 \mathcal{E}(p)-\sum_{L \in \mathrm{L}} \mathcal{E}\left(p-p_{L}\right)=h^{2} \delta_{h}(p),
$$

equation (14) yields

$$
v_{1}(p)=u_{1}+\left(u_{0}-u_{1}\right) \frac{\mathcal{E}(p)-\mathcal{E}(R)}{\frac{1-k}{4 k}-\mathcal{E}(R)}, \quad p \neq p_{0,0} .
$$


In this form, the solution of (9) is easily analyzed for asymptotic exactness. Indeed, taking into account (13) and (19), we get

$$
v_{1}(p)=u_{1}+\left(u_{0}-u_{1}\right) \frac{\ln \frac{R}{r}}{\frac{\pi / 2}{k}+\ln \frac{R}{h}+\frac{3}{2} \ln 2+\gamma-\frac{\pi}{2}}+O\left(\frac{h^{2}}{r^{2}}\right) .
$$

Thus, we arrived at (cf. (4))

Theorem. If the coefficient $k$ in (9) is chosen $k=\frac{\pi / 2}{\ln \frac{h}{\varepsilon}+\frac{\pi}{2}-\frac{3}{2} \ln 2-\gamma}$, then the difference scheme (9) asymptotically approximates the problem (1) - (3).

We come to the conclusion that the value for $k$ used in the literature, $k=$ $\frac{\pi / 2}{\ln \frac{h}{\varepsilon}}$, results in a difference scheme that is not asymptotically exact. In fact, for this $k$, the solution of (9) can be rewritten in the form

$$
v_{1}(p)=u(r)+e_{1}+O\left(\frac{h^{2}}{r^{2}}\right),
$$

where $u(r)$ is defined by (4), and

$$
e_{1}=-\frac{\left(u_{0}-u_{1}\right) \ln \left(\frac{R}{r}\right)}{\ln \left(\frac{R}{\varepsilon}\right)}\left(\frac{\frac{3}{2} \ln 2+\gamma-\frac{\pi}{2}}{\ln \frac{R}{\varepsilon}+\frac{3}{2} \ln 2+\gamma-\frac{\pi}{2}}\right),
$$

is non-removable asymptotical error $\left(\frac{3}{2} \ln 2+\gamma-\frac{\pi}{2} \approx 4.614 \cdot 10^{-2}\right)$. It can be shown using results from [21] that difference scheme (9) from [4],[8],[9] is the same as from [2],[5]-[7], and [10]. Consequently, the latter do not provide an asymptotical approximation (in a mathematical sense) either.

Remark. Analysis of the above approximation \#1 can be done differently using the techniques found in [20] (see Appendix B). However that approach does not extend to the approximation \#2.

\subsection{Approximation \#2 (Analysis)}

Let us seek the solution of (9) in the form

$$
v_{2}(p)=\alpha_{2}+\beta_{2} \mathcal{E}(p)+\gamma_{2} \sum_{L \in \mathbf{L}} \mathcal{E}\left(p-p_{L}\right)+\mu_{2} \sum_{F \in \mathbf{F}} \mathcal{E}\left(p-p_{F}\right),
$$

Applying the operator $\Lambda_{2}$ and the corresponding boundary conditions to (22), one gets the system of equations 


$$
\begin{aligned}
&\left(\Lambda_{2} v_{2}(p)\right)_{(1)} \quad \equiv-\gamma_{2}+\beta_{2}\left(N_{L} \mathcal{E}(p)\right)_{(1)}+\gamma_{2} \sum_{L \in \mathrm{L}}\left(N_{L} \mathcal{E}\left(p-p_{L}\right)\right)_{(1)} \\
& \quad+\mu_{2} \sum_{F \in \mathrm{F}}\left(N_{L} \mathcal{E}\left(p-p_{F}\right)\right)_{(1)}=0 \\
&\left(\Lambda_{2} v_{2}(p)\right)_{(2)} \equiv-\mu_{2}+\beta_{2}\left(B_{F} \mathcal{E}(p)\right)_{(2)}+\gamma_{2} \sum_{L \in \mathrm{L}}\left(B_{F} \mathcal{E}\left(p-p_{L}\right)\right)_{(2)} \\
&+\mu \sum_{F \in \mathrm{F}}\left(B_{F} \mathcal{E}\left(p-p_{F}\right)\right)_{(2)}=0, \\
& \alpha_{2}+4 \gamma_{2} \mathcal{E}_{10}+4 \mu_{2} \mathcal{E}_{11}=u_{0}, \\
& \alpha_{2}+\left(\beta_{2}+4 \gamma_{2}+4 \mu_{2}\right) \mathcal{E}(R)=u_{1} .
\end{aligned}
$$

The explicit expressions for terms $\left(N_{L} \mathcal{E}(p)\right)_{(1)},\left(N_{L} \mathcal{E}\left(p-p_{L}\right)\right)_{(1)}, \quad\left(N_{L} \mathcal{E}(p-\right.$ $\left.\left.p_{F}\right)\right)_{(1)},\left(B_{F} \mathcal{E}(p)\right)_{(2)},\left(B_{F} \mathcal{E}\left(p-p_{L}\right)\right)_{(2)}$ and $\left(B_{F} \mathcal{E}\left(p-p_{F}\right)\right)_{(2)}$ can be written as

$$
\begin{aligned}
& \left(N_{L} \mathcal{E}(p)\right)_{(1)}=(k-1) \mathcal{E}_{00}+(1-k) \mathcal{E}_{10} \\
& +2(1-b) \mathcal{E}_{10}+2(b-1) \mathcal{E}_{11}, \\
& \sum_{L \in \mathrm{L}}\left(N_{L} \mathcal{E}\left(p-p_{L}\right)\right)_{(1)}=(k-1) \cdot 4 \mathcal{E}_{10}+(1-k)\left(2 \mathcal{E}_{11}+\mathcal{E}_{20}+\mathcal{E}_{00}\right) \\
& +2\left((b-1)\left(2 \mathcal{E}_{10}+2 \mathcal{E}_{21}\right)+(1-b)\left(2 \mathcal{E}_{10}+2 \mathcal{E}_{20}+\mathcal{E}_{00}\right)\right) \\
& \sum_{F \in \mathrm{F}}\left(N_{L} \mathcal{E}\left(p-p_{F}\right)\right)_{(1)}=(k-1) \cdot 4 \mathcal{E}_{11}+(1-k)\left(2 \mathcal{E}_{21}+2 \mathcal{E}_{10}\right) \\
& +2\left((b-1)\left(\mathcal{E}_{00}+\mathcal{E}_{22}+2 \mathcal{E}_{20}\right)+(1-b)\left(2 \mathcal{E}_{10}+2 \mathcal{E}_{21}\right)\right) \\
& \left(B_{F} \mathcal{E}(p)\right)_{(2)}=2\left((b-1) \mathcal{E}_{10}+(1-b) \mathcal{E}_{11}\right) \\
& \sum_{L \in \mathrm{L}}\left(B_{F} \mathcal{E}\left(p-p_{L}\right)\right)_{(2)} \\
& =2\left((b-1)\left(2 \mathcal{E}_{11}+\mathcal{E}_{20}+\mathcal{E}_{00}\right)+(1-b)\left(2 \mathcal{E}_{10}+2 \mathcal{E}_{21}\right)\right) \\
& \sum_{F \in \mathrm{F}}\left(B_{F} \mathcal{E}\left(p-p_{F}\right)\right)_{(2)} \\
& =2\left((b-1)\left(2 \mathcal{E}_{21}+2 \mathcal{E}_{10}\right)+(1-b)\left(\mathcal{E}_{22}+2 \mathcal{E}_{20}+\mathcal{E}_{00}\right)\right)
\end{aligned}
$$


The solution to the system (23) is

$$
\begin{array}{r}
\alpha_{2}=u_{1}-\left(u_{0}-u_{1}\right) \frac{\mathcal{E}(R)}{\frac{1-k}{4 k}-\mathcal{E}(R)-t}, \\
\beta_{2}=\left(u_{0}-u_{1}\right) \frac{\frac{1}{k}}{\frac{1-k}{4 k}-\mathcal{E}(R)-t}, \\
\gamma_{2}=\left(u_{0}-u_{1}\right) \frac{\frac{k-1}{4 k}-\frac{t}{\frac{4}{\pi}-1}}{\frac{1-k}{4 k}-\mathcal{E}(R)-t}, \\
\mu_{2}=\left(u_{0}-u_{1}\right) \frac{\frac{t}{\frac{4}{\pi}-1}}{\frac{1-k}{4 k}-\mathcal{E}(R)-t},
\end{array}
$$

where $t=\frac{(b-1)\left(\frac{4}{\pi}-1\right)^{2}}{2+2(b-1)\left(\frac{64}{3 \pi}-6\right)}$.

Finally the solution of the difference scheme (11) has the form

$$
v_{2}(p)=u_{1}+\left(u_{0}-u_{1}\right) \frac{\mathcal{E}(p)-\mathcal{E}(R)+\frac{t}{\frac{4}{\pi}-1}\left(\sum_{F \in \mathrm{F}} \mathcal{E}\left(p-p_{F}\right)-\sum_{L \in \mathrm{L}} \mathcal{E}\left(p-p_{L}\right)\right)}{\frac{1-k}{4 k}-\mathcal{E}(R)-t} .
$$

For the values $k=\frac{\pi / 2}{\ln \frac{h}{\varepsilon}}$ and $b=\frac{2 \arctan \frac{1}{2}}{\ln 2}$ used in [4], [8], [9], we get $t \approx$ $9.952 \cdot 10^{-3}$ and $\frac{3}{2} \ln 2+\gamma-\frac{\pi}{2}-2 \pi t \approx-1.6392 \cdot 10^{-2}$.

The asymptotical expansion of $v_{2}(p)$ is

$$
v_{2}(p)=u_{1}+\left(u_{0}-u_{1}\right) \frac{\ln \frac{R}{r}}{\ln \frac{R}{\varepsilon}+\frac{3}{2} \ln 2+\gamma-\frac{\pi}{2}-2 \pi t}+O\left(\frac{h^{2}}{r^{2}}\right)
$$

or (see (4)),

$$
v_{2}(p)=u(r)+e_{2}+O\left(\frac{h^{2}}{r^{2}}\right)
$$

where

$$
e_{2}=-\frac{\left(u_{0}-u_{1}\right) \ln \frac{R}{r}}{\ln \frac{R}{\varepsilon}}\left(\frac{\frac{3}{2} \ln 2+\gamma-\frac{\pi}{2}-2 \pi t}{\ln \frac{R}{\varepsilon}+\frac{3}{2} \ln 2+\gamma-\frac{\pi}{2}-2 \pi t}\right)
$$

It follows from (24) that the difference scheme (11) is more accurate than the scheme (9), but it has the non-removable asymptotical error $e_{2}$ as well. 


\section{Acknowledgements}

The authors are deeply grateful to V. B. Andreev for suggesting the problem and for useful advice.

\section{References}

[1] A. A. Samarskii, On the effect of fixing on the characteristic frequencies of closed volumes. Dokl. Akad. Nauk USSR, 63, No.6 (1948), 631-634.

[2] D. W. Peaceman, Interpretation of well-block pressures in numerical reservoir simulation (includes associated paper 6988). SPE Journal, 18, No.3 (June 1978), 183-194.

[3] K. Aziz, A. Settari, Petroleum reservoir simulation. Applied Science Publishers, London, (1979).

[4] M. G. Alishaev, G. O. Khazamov, The use of matrix pivotal condensation for problem of oil-field flooding. USSR Computation Mathematics and Mathematical Physics, 24, No.2 (1984), 44-51.

[5] H. K. van Poollen, E. A. Breitenbach, D. H. Thurnau, Treatment of individual wells and grids in reservoir modeling SPE Journal, 8, No.4 (Dec. 1968), $341-346$.

[6] J. Kuniansky, J. G. Hillestad, Reservoir simulation using bottomhole pressure boundary conditions. SPE Journal, 20, No.6 (Dec. 1980), 473-486.

[7] A. S. Williamson, J. E. Chappelear, Representing wells in numerical reservoir simulation: Part 1 - Theory. SPE Journal, 21, No.3 (June 1981), $323-338$.

[8] A. N. Chekalin, Numerical solutions of filtration problems in water-oil fields. Kazan University, Kazan, USSR, (1982) (in Russian).

[9] G. G. Vakhitov, Difference methods for solving oil-field development problems. Nedra, Leningrad, USSR (1971) (in Russian).

[10] K. Schwabe, J. Brandt, Prediction of reservoir behavior using numerical simulators. paper SPE 1857 presented at the SPE-AIME 42-nd Annual Fall Meeting, Houston, Oct. 1-4, (1967). 
[11] C. C. Mattax, R. L. Dalton, Reservoir Simulation, SPE Monograph, volume 13 (1990).

[12] T. Ertekin, J. H. Abou-Kassem, G. R. King, Basic Applied Reservoir Simulation, SPE Textbook, volume 10 (2001).

[13] J. Fanchi, Principles of Applied Reservoir Simulation, 3-rd Edition, Elsevier GPP (2006).

[14] M. Carlson, Practical Reservoir Simulation, PennWell Corporation (2006).

[15] E. Holstein (Editor), Petroleum Engineering Handbook, Reservoir Engineering, volume V(b), Chapt.17 (2007).

[16] S. L. Sobolev, On a difference equation. Dokl. Akad. Nauk USSR, 87, No.3 (1952), 341-343.

[17] R. J. Duffin, D. H. Shaffer, Asymptotic expansion of double Fourier transforms. Duke Math. Journal, 27, No.4 (1960), 581-596.

[18] W. H. McCrea, F. J. W. Whipple, Random paths in two and three dimensions. Proceedings of Royal Soc. Edinburgh, 60, No.3 (1939-1940), 281-298.

[19] A. Stöhr, Über einige lineare partielle Differenzgleichungen mit konstanten Koeffizienten. Math. Nachr., 3, No.6 (1949-1950), 330-357.

[20] V. B. Andreev, S. A. Kryakvina, The source function of the difference Laplace operator. USSR Computational Mathematics and Mathematical Physics, 12, Issue 2 (1972), 101-114.

[21] N. V. Parshkova, Examination of the finite-element method in approximation of the borehole problem. (English) Moscow University Computational Mathematics and Cybernetics, No.3, (1985), 17-25; translation from Vestnik Moskovskogo Universiteta Ser. XV, No.3, (1985), 15-21.

\section{A. Derivation of the Approximations \#1 and \#2 Based on Consideration of Fluxes}

In this Appendix, we expose the common structure of numerous derivations found in various applied journals (see, for example, [8],[9]). These derivations are written in a professional jargon. Our objective is to make those derivations readable for a mathematician. 
Let $u=u(r)$ be the solution of (1) - (3) given by (4). By Green's second formula applied to (1), we have

$$
\oint_{\Gamma} \frac{\partial u}{\partial n} d \Gamma=0 .
$$

for any contour $\Gamma$ not enclosing the well. We will arrive at the equations (9) and then at equations (11) by expressing the discrete version of (25) for all $h \times h$ square contours centered at grid points in the interior of $\omega$. For a grid point $p_{i j}$ not too close to $(0,0)$, i.e. to the well, one gets $\Lambda u_{i j}=0$, whereas accounting for the flux out of the well at points near the well leads to a modified expression for $\Lambda$. The modification depends on which "points near the well" are taken into consideration. We present two such modifications, which lead respectively to (9) with $k=\frac{\pi / 2}{\ln \frac{h}{\varepsilon}}$ and (11) with $k=\frac{\pi / 2}{\ln \frac{h}{\varepsilon}}, b=\frac{2 \arctan \frac{1}{2}}{\ln 2}$.

For a generic $(i, j) \in \omega$ "away" from $(0,0)$, the equality to zero of the outward flux of $\nabla u$ through the boundary of the $h \times h$ square centered at $p_{i, j}$ amounts to $\Lambda u_{i, j}=0$.

\section{A.1. Derivation of the Approximation \#1}

Let us modify the discretized (25) for the four $h \times h$ squares centered at $p_{L}, L \in$ $\mathrm{L}$ (and only for those). Namely, for each $L$, the side of the square facing the well will be ascribed the flux value produced by the exact (analytic) solution $u(r)$.

The analytic solution $u(r)$ of (1) - (3) is given by (4). So, the outward flux $Q$ of $\nabla u$ through a circle of an arbitrary radius $\rho$ centered at $(0,0)$ is

$$
Q=\int_{0}^{2 \pi} u^{\prime}(\rho) \rho d \varphi=-\frac{2 \pi}{\ln \frac{R}{\varepsilon}}\left(u_{0}-u_{1}\right) .
$$

The outward flux of $\nabla u$ is the same through any contour enclosing $(0,0)$, in particular, through the square $A B C D$ in Fig.1. Due to rotational symmetry, the flux of $\nabla u$ through each side of $A B C D$ is $Q / 4$.

Because of the symmetry, we discretize (25) only for the square centered at $p_{0,1}$ (the square $A B E F$ in Fig.1). With the terms following the order of the sides $B E, E F, F B, B A$, we have (cf. (??))

$$
h\left(u_{1,1}-u_{1,0}\right) \frac{1}{h}+h\left(u_{2,0}-u_{1,0}\right) \frac{1}{h}+h\left(u_{1,-1}-u_{1,0}\right) \frac{1}{h}-Q / 4=0 .
$$




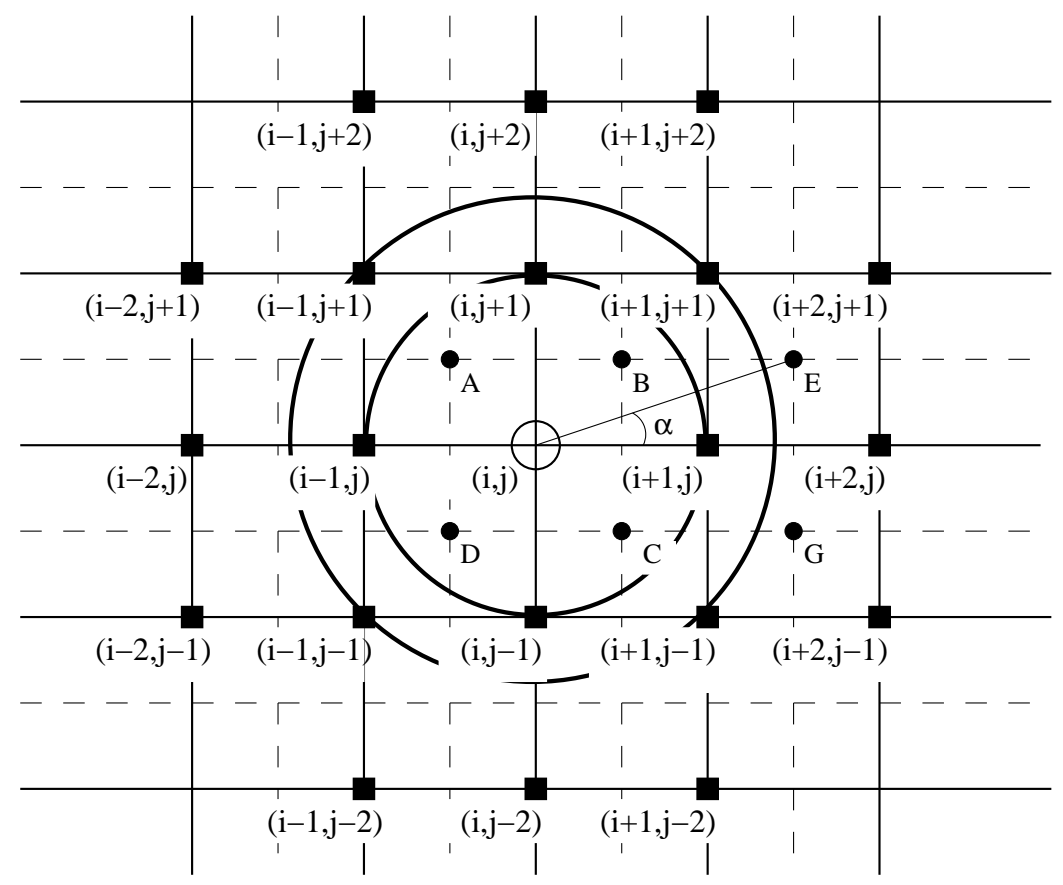

Figure 1: Contours of filtration near the well

To "discretize" the $Q / 4$ term, we use (26), identify $u_{0}$ with $u_{0,0}$, and notice that $u_{1,0}=u(h)=u_{1}+\left(u_{0}-u_{1}\right) \frac{\ln \frac{R}{\varepsilon}}{\ln \frac{h}{\varepsilon}}$, which allows to express $u_{1}$ through $u_{1,0}$ :

$$
u_{1}=\frac{\ln \frac{R}{\varepsilon}}{\ln \frac{h}{\varepsilon}}\left(u_{1,0}-\frac{\ln \frac{R}{h}}{\ln \frac{R}{\varepsilon}} u_{1,0}\right) .
$$

Thus, from (26), $u_{0}=u_{0,0}$, and (28),

$$
-Q / 4=k\left(u_{0,0}-u_{1,0}\right), \quad k=\frac{\pi / 2}{\ln \frac{h}{\varepsilon}}
$$

and (27) becomes equivalent to (8).

Similarly, the modified "zero-flux statement" for the $h \times h$ squares centered at $p_{L}, L=(0,1),(-1,0),(0,-1)$, becomes $\Lambda_{1} u_{L}=0$. If, for every $(i, j) \notin \mathbf{L}$, we put $\Lambda_{1} u_{i, j}=\Lambda u_{i, j}$, then we arrive at (9) (wth $\left.k=\frac{\pi / 2}{\ln \frac{h}{\varepsilon}}\right)$. 


\section{A.2. Derivation of the Approximation \#2}

This time, we modify the discretization of (25) for the eight $h \times h$ squares centered at $p_{L}, L \in \mathrm{L}$, and $p_{F}, F \in \mathrm{F}$ (and only for those). For these squares, some of their sides will be ascribed the flux values produced by the analytic solution $u(r)$.

Because of the symmetry, we illustrate the modifications for one of the $L$-points, $(1,0)$, and one of the $F$-points, $(1,1)$.

For $(1,0)$, the discretization of $(25)$ is

$$
Q_{A B}+Q_{B E}+\left(u_{2,0}-u_{1,0}\right)+Q_{A F}=0 .
$$

Evidently, $Q_{A B}=-Q / 4=k\left(u_{0,0}-u_{1,0}\right)$ (see $\left.(29)\right)$. To express $Q_{B E}$ through $u_{1,1}$ and $u_{1,0}$, notice that

$$
Q_{B E}=\frac{\frac{\pi}{4}-\alpha}{2 \pi} Q=-\frac{\arctan \frac{1}{2}}{\ln \frac{R}{\varepsilon}}\left(u_{0}-u_{1}\right)
$$

(see (26) for the second equality) and use

$u_{1,0}=u(h)=u_{1}+\left(u_{0}-u_{1}\right) \frac{\ln \frac{R}{h}}{\ln \frac{R}{\varepsilon}}$

and $u_{1,1}=u(h \sqrt{2})=u_{1}+\left(u_{0}-u_{1}\right) \frac{\ln \frac{R}{h \sqrt{2}}}{\ln \frac{R}{\varepsilon}}$ to find that

$\left(u_{0}-u_{1}\right)=\frac{\ln \frac{R}{\varepsilon}}{\ln \sqrt{2}}\left(u_{1,0}-u_{1,1}\right)$, whence $(31)$ gives

$$
Q_{B E}=b\left(u_{1,1}-u_{1,0}\right), \quad b=\frac{2 \arctan \frac{1}{2}}{\ln 2} .
$$

Similarly, one arrives at $Q_{A F}=b\left(u_{1,-1}-u_{1,0}\right)$ and $(30)$ becomes

$$
k\left(u_{0,0}-u_{1,0}\right)+b\left(u_{1,1}-u_{1,0}\right)+\left(u_{2,0}-u_{1,0}\right)+b\left(u_{1,-1}-u_{1,0}\right) .
$$

Since the left hand side of (33) can be written as $h^{2} \Lambda_{2} u_{1,0}$, then (33) becomes $\Lambda_{2} u_{1,0}=0$.

Similarly, the modified "zero-flux statement" for the $h \times h$ squares centered at $p_{L}, L=(0,1),(-1,0),(0,-1)$, become $\Lambda_{2} u_{L}=0$.

For $(1,1)$, the discretization of $(25)$ is

$$
-Q_{B E}+\left(u_{2,1}-u_{1,1}\right)+\left(u_{1,2}-u_{1,1}\right)+Q_{B H}=0
$$

$\left(Q_{B E}\right.$ was introduced as part of the outward flux for $\left.A B E F\right)$. Just as $Q_{B E}$ has an expression through $u_{1,1}$ and $u_{1,0}, Q_{B H}$ can be expressed through $u_{1,1}$ and $u_{0,1}: Q_{B H}=b\left(u_{0,1}-u_{1,1}\right)$. Thus, (34) becomes

$$
b\left(u_{1,0}-u_{1,1}\right)+\left(u_{2,1}-u_{1,1}\right)+\left(u_{1,2}-u_{1,1}\right)+b\left(u_{0,1}-u_{1,1}\right)=0,
$$


which is the same as $\Lambda_{2} u_{1,1}=0$. Similarly, the modified "zero-flux statement" for the $h \times h$ squares centered at $p_{F}, F=(-1,1),(-1,-1),(1,-1)$, become $\Lambda_{2} u_{F}=0$. Finally, if for every $(i, j) \notin \mathrm{L} \cup \mathrm{F}$, we put $\Lambda_{2} u_{i, j}=\Lambda u_{i, j}$, then $\Lambda_{2} u_{i, j}=0$ for all $(i, j)$, and we arrived at (11).

\section{A.3. Derivation of the Approximations \#1 and \#2 Based on Explicit Isolation of Singularity}

The solution of the differential problem (1) - (3) is considered in the following form:

$$
u(x, y)=\bar{u}(x, y)+S .
$$

$S$ is the singularity, expressed by

$$
S= \begin{cases}\mu \ln r & \text { for }(x, y) \in D, \\ 0 & \text { for }(x, y) \in C D,\end{cases}
$$

where $\mu=$ const and $D=\left\{(x, y) \mid\left(x^{2}+y^{2}\right)^{1 / 2}=r<\rho\right\}$. This formulation implies that $\bar{u}(x, y)$ does not have a logarithmic singularity, but it is discontinuous on the boundary of the domain $D$. Therefore,

$$
\begin{aligned}
& \Delta \bar{u}(x, y)=0, \text { for all }(x, y) \notin \partial D, \\
& {\left[\frac{\partial \bar{u}}{\partial n}\right]_{\partial D}=\left.\mu \frac{\partial \ln r}{\partial n}\right|_{\partial D},} \\
& {[u]_{\partial D}=\mu \ln \rho .}
\end{aligned}
$$

Here brackets denote the 'jump' of a function across the boundary $\partial D$ from inside out.

In what follows, we refer to the position of the well as $(i, j)$ instead of $(0,0)$. This makes the exposition more readable.

From (36) we have

$$
\left(\frac{\partial u}{\partial x}\right)_{i+\frac{1}{2}, j}=\left(\frac{\partial \bar{u}}{\partial x}\right)_{i+\frac{1}{2}, j}+\mu \frac{\frac{1}{2} h}{\frac{1}{4} h^{2}+(y-j h)^{2}},
$$

From these equations it follows that $\mu$ is now:

$$
\mu=\frac{u_{i+1, j}-u_{0}}{\ln \frac{h}{\varepsilon}}-\frac{\bar{u}_{i+1, j}-\bar{u}_{i, j}}{\ln \frac{h}{\varepsilon}} .
$$


Note that

$$
\int_{y_{j}-\frac{1}{2} h}^{y_{j}+\frac{1}{2} h} \frac{\frac{1}{2} h}{\frac{1}{4} h^{2}+(y-j h)^{2}} d y=\frac{\pi}{2} .
$$

Let $\rho=h$. Then, taking into account (36) and using (37) - (39), the difference analog of (25) can be expressed by

$$
\begin{aligned}
\left(v_{i+2, j}-v_{i+1, j}\right) \quad & +\frac{\pi / 2}{\ln \frac{h}{\varepsilon}}\left(u_{0}-v_{i+1, j}\right) \\
& +\left(v_{i+1, j+1}-v_{i+1, j}\right)+\left(v_{i+1, j-1}-v_{i+1, j}\right)=0 .
\end{aligned}
$$

(Compare to (9).)

To illustrate the second more complicated approximation, let $\rho=h \sqrt{2}$. Recall that the second approximation uses not only the the block corresponding to the radius $r=h$, but also the blocks describing the fluxes corresponding to the radius $r=h \sqrt{2}$. The following formulae for the derivatives in terms of equation (25) must be added to our consideration:

$$
\begin{aligned}
\left(\frac{\partial u}{\partial y}\right)_{i, j \pm \frac{1}{2}} & =\left(\frac{\partial \bar{u}}{\partial y}\right)_{i, j \pm \frac{1}{2}}+\mu \frac{\frac{1}{2} h}{(x-i h)^{2}+\frac{1}{4} h^{2}}=\left(\frac{\partial \bar{u}}{\partial y}\right)_{i, j \pm \frac{1}{2}} \\
& +\frac{\frac{1}{2} h}{(x-i h)^{2}+\frac{1}{4} h^{2}}\left( \pm \frac{u_{i, j \pm 1}-u_{0}}{\ln \sqrt{2}} \mp \frac{\bar{u}_{i, j \pm 1}-\bar{u}_{i, j}}{\ln \sqrt{2}}\right) .
\end{aligned}
$$

Since

$$
\int_{x_{i}-\frac{1}{2} h}^{x_{i}+\frac{1}{2} h} \frac{\frac{1}{2} h}{\frac{1}{4} h^{2}+(x-i h)^{2}} d x=\arctan \frac{1}{2},
$$

it can be shown that for contour $B E G C B$, the difference analog of (25) is

$$
\begin{aligned}
\left(v_{i+2, j}-v_{i+1, j}\right)-\quad & \frac{\pi / 2}{\ln \frac{h}{\varepsilon}}\left(v_{i+1, j}-u_{0}\right)+\frac{2 \arctan \frac{1}{2}}{\ln 2}\left(v_{i+1, j+1}-v_{i+1, j}\right) \\
& -\frac{2 \arctan \frac{1}{2}}{\ln 2}\left(v_{i+1, j}-v_{i+1, j-1}\right)=0 .
\end{aligned}
$$

This difference analog can be computed similarly for all other nodes lying on the contour corresponding $\rho=h \sqrt{2}$. 


\section{B. Alternative Analysis of Approximation \#1}

Let us consider one more approximation of the BVP (1) - (3).

$$
\begin{array}{r}
\Lambda v(p)-q \cdot\left(v(p)-u_{0}\right) \delta_{h}(p)=0, \quad(x, y) \in \omega, \\
v(p)=u(p), \quad(x, y) \in \Omega \backslash \omega .
\end{array}
$$

Here $q$ is a parameter.

The solution of the problem (40) is found in [20]:

$$
\begin{aligned}
& v(p)=u_{1}-\frac{q \cdot\left(u_{0}-u_{1}\right)}{1+\frac{q}{2 \pi}\left(\ln \frac{R}{h}+\frac{3}{2} \ln 2+\gamma\right)}\left(\mathcal{E}(p)-\frac{\ln \frac{h}{R}-\frac{3}{2} \ln 2-\gamma}{2 \pi}\right) \\
& +O\left(\frac{h^{2}}{r^{2}}\right) .
\end{aligned}
$$

Also it is shown in [20] that if

$$
q=\frac{2 \pi}{\ln \left(\frac{h}{\varepsilon}\right)-\frac{3}{2} \ln 2-\gamma},
$$

then the solution is

$$
v(p)=u_{1}+\frac{\left(u_{0}-u_{1}\right) \ln \left(\frac{R}{r}\right)}{\ln \left(\frac{R}{\varepsilon}\right)}+O\left(\frac{h^{2}}{r^{2}}\right) .
$$

In other words, solution (44) asymptotically approximates the exact analytical solution (4) of problem (1) - (3).

Let us show that the approximation (9) can be rewritten in the form (40) and, in particular, express $q$ through $k$.

Let us write down difference equations (9) for one of nodes from the set $\mathbf{L}$. For example for $(1,0)$

$$
\left(v_{2,0}-v_{1,0}\right)-k\left(v_{1,0}-u_{0,0}\right)+\left(v_{1,1}-v_{1,0}\right)-\left(v_{1,0}-v_{1,-1}\right)=0
$$

and difference equations (40) for the same node:

$$
\left(v_{2,0}-v_{1,0}\right)-\left(v_{1,0}-v_{0,0}\right)+\left(v_{1,1}-v_{1,0}\right)-\left(v_{1,0}-v_{1,-1}\right)=0 .
$$

We find $v_{0,0}$ from equation (40) written for node $(0,0)$ :

$$
4\left(v_{1,0}-v_{0,0}\right)-q\left(v_{0,0}-u_{0}\right)=0,
$$


and it follows that

$$
v_{0,0}=\frac{4 v_{1,0}}{q+4}+\frac{q u_{0}}{q+4} .
$$

Subtracting (45) from (46) then substituting (47) we find

$$
k\left(v_{1,0}-u_{0}\right)=v_{1,0}-v_{0,0}=v_{1,0}-\frac{4 v_{1,0}}{q+4}-\frac{q u_{0}}{q+4}=\frac{q}{q+4}\left(v_{1,0}-u_{0}\right) .
$$

Thus, we have

$$
k=\frac{q}{q+4}, \quad \text { or } \quad q=\frac{4 k}{1-k} .
$$

To the 'engineering' value $k=\frac{\pi / 2}{\ln \frac{h}{\varepsilon}}$ there corresponds $q=\frac{2 \pi}{\ln \frac{h}{\varepsilon}-\frac{\pi}{2}}$, which differs from the $q$ value given by (43). Therefore, the approximation \#1 with $k=\frac{\pi / 2}{\ln \frac{h}{\varepsilon}}$ is not asymptotically exact.

Conversely, to $q$ given by (43), there corresponds $k=\frac{\pi / 2}{\ln \frac{h}{\varepsilon}+\left(\pi / 2-\frac{3}{2} \ln 2-\gamma\right)}$, and the approximation \# 1 with this $k$ is asymptotically exact. Thus, we have re-derived the Theorem from Sec. 2.2. 